\title{
EXPERIMENTAL CONTROL OF THE NUMBER OF IONIZED DONORS IN AN AlGaAs/GaAs HETEROSTRUCTURE
}

\author{
P. Denk, T. Schlösser, K. Ensslin \\ Sektion Physik, LMU München, 80539 München, Germany \\ AND M. HOLLAND \\ Dept. of Electronics, University of Glasgow, United Kingdom
}

\begin{abstract}
The high mobility of electrons in $\mathrm{AlGaAs}-\mathrm{GaAs}$ heterostructures relies on the concept of modulation doping. As a sample is cooled down to $T=$ $4.2 \mathrm{~K}$ under a fixed gate bias the number of ionized donors can be frozen and is then independent on the gate potential. We discuss the consequences of this procedure on the electron density and mobility in a two-dimensional electron gas. For a laterally patterned sample we find that the amplitude of the potential modulation can be maximized for a given carrier density by a suitably chosen cool-down voltage.
\end{abstract}

PACS numbers: 73.20.Dx, 03.65.Sq, 73.50.Jt

The invention of modulation doping [1] has lead to the realization of ultrahigh electron mobilities in AlGaAs-GaAs heterostructures [2]. At low temperatures the mobility is usually limited by Coulomb scattering of the electrons at the residual impurities in the GaAs channel as well as at the smooth potential perturbations arising from the ionized donors in the intentionally doped region above the spacer layer. The electron density can be calculated self-consistently [3] from the layer sequence and the number of ionized donors which depends sensitively on the position of the Fermi energy at room temperature. If the Fermi energy is changed at low temperatures the thermal energy of the electrons is too low to overcome the AlGaAs barrier between the electron channel and the doped region leading to a fixed degree of ionization. The carrier density of the electrons, however, can still be tuned with an external gate voltage at low temperatures because the number of ionized donors is fixed. The method to cool down a sample under a fixed gate voltage $V_{\text {cd }}$ can therefore be used to realize different and fixed values of ionized donors at low temperatures keeping all other sample parameters the same.

The possible correlation of ionized donors and the impact on the mobility of the electron gas was investigated by Buks et al. [4]. Here we are rather interested in the possibility to influence the potential and with it the carrier density at a 
given gate voltage at low temperatures. In particular we find that the number of ionized donors can be modified by a factor of two for suitably chosen cool-down voltages. Furthermore we show that the controlled freezing of donors can be used to optimize the potential modulation of a lateral superlattice.

The samples are grown by molecular beam epitaxy and have the following layer sequence on top of the substrate and the buffer layer: $20 \mathrm{~nm}$ undoped $\mathrm{Al}_{x} \mathrm{Ga}_{1-x} \mathrm{As}$ spacer, $12 \mathrm{~nm} \mathrm{Si}$ doped $\mathrm{Al}_{x} \mathrm{Ga}_{1-x} \mathrm{As}, 13 \mathrm{~nm}$ undoped $\mathrm{Al}_{x} \mathrm{Ga}_{1-x} \mathrm{As}$ and $9 \mathrm{~nm}$ undoped GaAs cap. The Al content is $x=0.3$ and the doping level is $3 \times 10^{18} \mathrm{~cm}^{-3}$ as calibrated on GaAs bulk samples. At $T=4.2 \mathrm{~K}$ the carrier density is $3.5 \times 10^{11} \mathrm{~cm}^{-2}$ and the mobility $500000 \mathrm{~cm}^{2} /(\mathrm{Vs})$. A gate electrode covering a Hall bar is evaporated enabling us to apply a front gate voltage with respect to the two-dimensional electron gas (2DEG).

At room temperature a finite gate voltage is applied and the sample is then cooled down under this fixed cool-down voltage $V_{c d}$ to a temperature of $T=$ $4.2 \mathrm{~K}$. At this low temperature the magnetoresistance as well as the Hall resistance is measured for different values of the gate voltage $V_{\mathbf{g}}$ in order to extract the carrier density and the mobility. The experimentally obtained carrier density as a function of gate voltage for several cool-down voltages is presented in Fig. 1 (solid symbols). The four curves are parallel to each other indicating the capacitor-like geometry between the front gate and the 2DEG. Above a carrier density of $N_{\mathrm{s}} \approx$ $4.5 \times 10^{11} \mathrm{~cm}^{-2}$ the curves saturate. The additional electrons induced by the gate voltage start to occupy states in the doped AlGaAs regions where the conduction band falls below the Fermi energy. The arising parallel conduction of the sample can be detected in the magnetoresistance.

In order to get a quantitative understanding of the change in the ionization degree of the donors we solved Schrödinger and Poisson equation self-consistently (see for example [3]) taking the layer thicknesses from the growth as input parameters. The only free parameter in the calculation is the number of ionized donors in the doped region. The open symbols in Fig. 1 represent results from this calculation for four values of ionized donor concentrations as indicated in the inset (in units of $10^{18} \mathrm{~cm}^{-3}$ ). The general agreement with the experimental data is excellent. The maximum number of ionized donors found from this analysis is $3.0 \times 10^{18} \mathrm{~cm}^{-3}$ in close agreement with the value used in the growth procedure. The minimum number is about half that value, namely $1.4 \times 10^{18} \mathrm{~cm}^{-3}$. In that case only the shallow donors are ionized representing about $50 \%$ of the total number of donors.

A superlattice of photoresist stripes is fabricated on the sample surface by electron beam lithography and a suitable development and exposure process. The pattern is transferred onto the 2DEG either by a carefully tuned wet etching step [5] or by a gate voltage which is applied to a front gate electrode that is evaporated on top of the patterned resist layer [6]. Both sample types lead to qualitatively similar results and we will restrict ourselves in the following to the discussion of the wet etched sample surface which was covered with a gate electrode after the photoresist has been taken off.

Figure 2 presents experimental data for the potential modulation as a function of carrier density as tuned by a front gate voltage. The different curves are obtained for different cool-down voltages $V_{\text {cd }}$. For current flow perpendicular to 


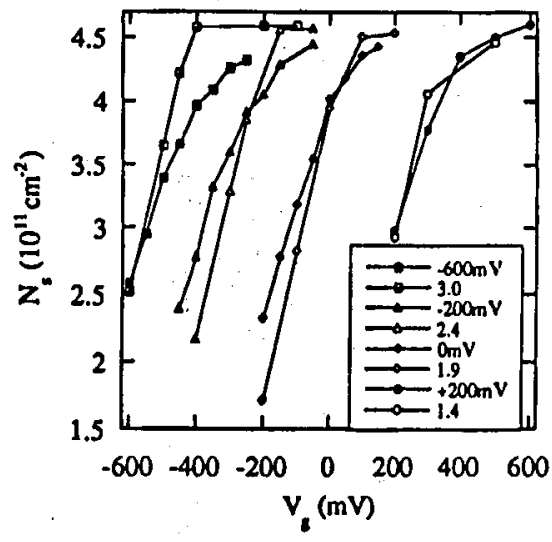

Fig. 1. Carrier density as extracted from low temperature Shubnikov-de Haas oscillations as a function of gate voltage for a laterally homogeneous $\mathrm{AlGaAs} / \mathrm{GaAs}$ heterostructure. The parameter in the experimental curves (solid symbols) is the cool-down voltage. The open symbols are obtained from a self-consistent calculation and the corresponding parameter is the density of ionized donors (in units of $10^{18} \mathrm{~cm}^{-3}$ ).

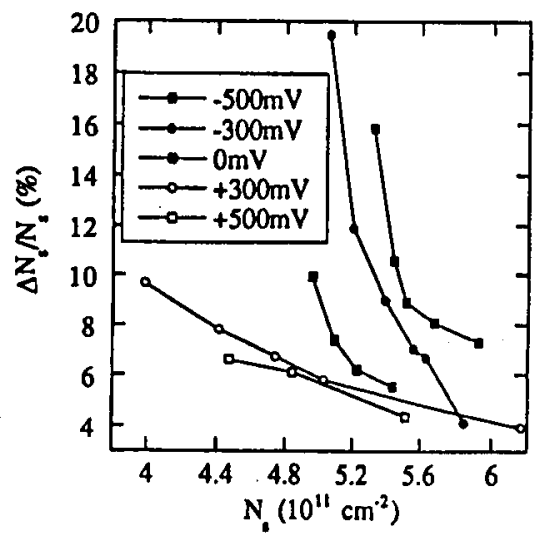

Fig. 2. Effective lateral modulation of the carrier density extracted from a theoretical analysis of commensurability oscillations as a function of normalized carrier density for a laterally patterned electron gas.

the potential modulation commensurability oscillations arise in the magnetoresistance [7] which can be used to extract the amplitude of the potential modulation [8]. The effective potential modulation in the plane of the 2DEG is found to be strongly reduced by screening effects [9].

For a sample surface patterned by wet etching the local carrier density is reduced under etched areas (see Fig. 3a). For a negative cool-down voltage $V_{c d}<0$ this leads to an enhanced and laterally more uniform density of ionized donors under the etched areas. Since the distance between the sample surface and the 


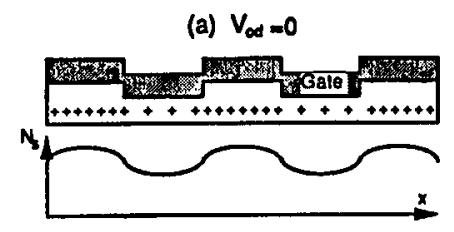

(b) $V_{\infty d}<0$

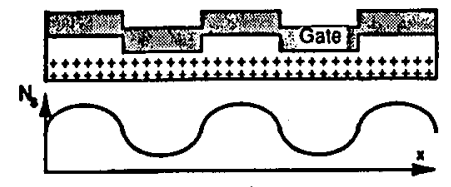

(c) $V_{\infty}>0$

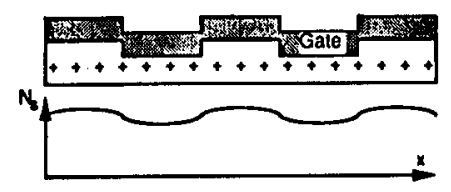

Fig. 3. Schematic of the sample structure for several cool-down voltages. The layer of crosses indicates the position of the ionized donors, the hatched area on top represents the gate metal and the amplitude of the $N_{s}$ versus $x$ curves gives an estimate for the effective potential modulation.

2DEG is reduced in the etched areas any applied gate voltage leads to a stronger electric field in those areas compared to the bare parts of the sample surface and therefore to a further increase in the potential modulation (see Fig. 3b). This explains the increase in $\Delta N_{\mathrm{s}} / N_{\mathrm{s}}$ as one goes from $V_{\mathrm{cd}}=0$ to $V_{\mathrm{cd}}=-300 \mathrm{mV}$ in Fig. 2. For a further increase in the negative cool-down voltage all donors will be ionized and the amplitude of the potential modulation at a given value of $N_{\mathrm{s}}$ will then saturate (see $V_{\mathrm{cd}}=-300 \mathrm{mV}$ to $-500 \mathrm{mV}$ in Fig. 2).

For positive cool-down voltages $V_{c d}>0$ one obtains a small and laterally uniform degree of ionization. In order to induce a given carrier density in the channel one has to apply a very positive gate bias that acts especially strong in the etched areas therefore compensating the original effect of the modulated sample surface. The effective potential modulation does not depend very strongly on the cool-down voltage (see $V_{\mathrm{cd}}=+300 \mathrm{mV}$ to $+500 \mathrm{mV}$ in Fig. 2, schematically in Fig. 3c). At least qualitatively we can understand the experimental observations as summarized in Fig. 2 by those straightforward considerations. The laterally distributed ionized donors are able to enhance or weaken the potential modulation depending on the sign of the cool-down voltage. We therefore have a means to realize a desired amplitude of the potential modulation by a well chosen cool-down voltage.

We are grateful to the Deutsche Forschungsgemeinschaft and the ESPRIT Basic Research Action for financial support. 


\section{References}

[1] H.L. Störmer, A.C. Gossard, W. Wiegmann, Solid State Commun. 41, 707 (1982).

[2] L.N. Pfeiffer, K.W. West, H.L. Störmer, K. Baldwin, Appl. Phys. Lett. 55, 1888 (1989).

[3] F. Stern, S. Das Sarma, Phys. Rev. B 30, 840 (1984).

[4] E. Buks, M. Heiblum, Y. Levinson, H. Shtrikman, Semicond. Sci. Technol. 9, 2031 (1994).

[5] R. Schuster, K. Ensslin, D. Wharam, S. Kühn, J.P. Kotthaus, G. Böhm, W. Klein, G. Tränkle, G. Weimann, Phys. Rev. B 49, 8510 (1994).

[6] T. Schlösser, K. Ensslin, F. Claro, J. P. Kotthaus, M. Holland, R. Ketzmerick, Phys. Rev. B 51, 10737 (1995).

[7] D. Weiss, K. v. Klitzing, K. Ploog, G. Weimann, Europhys. Lett. 8, 179 (1989).

[8] A.K. Geim, R. Taboryski, A. Kristensen, S.V. Dubonos, P.E. Lindelof, Phys. Rev. $B$ 46, 4324 (1992).

[9] J.P. Kotthaus, D. Heitmann, Surf. Sci. 113, 481 (1982). 